\title{
Age of Information of One-Hop Broadcast Communications in a CSMA Network
}

\author{
Andrea Baiocchi, Member, IEEE, and Ion Turcanu, Member, IEEE
}

\begin{abstract}
We consider a network of agents that send periodic updates to their neighbors. A trade-off between load on the shared communication channel and data timeliness is obtained by looking at the Age of Information (AoI) metric. We develop a model of a Carrier-Sense Multiple Access (CSMA) network with partial sensing, to calculate the AoI of one-hop broadcast messages exchanged among the agents. The model is applied to beacon messages in a vehicular network to gain insight into the impact of system parameters.
\end{abstract}

Index Terms-Age of Information, CSMA Networks, Vehicular Networks, Partial Sensing.

\section{INTRODUCTION}

The advent of the Internet of Things (IoT) makes networks of peer-agents sending broadcast update messages to one another a typical scenario. Vehicular Ad Hoc Networks (VANETs) are among the major examples of this communication paradigm, where vehicles exchange one-hop cooperative awareness messages to enable safety applications by maintaining an updated and extended view of the surrounding environment. Dedicated Short-Range Communication (DSRC) - currently the most mature vehicular communication technology - uses IEEE 802.11p PHY and MAC layers, based on the Carrier-Sense Multiple Access (CSMA) mechanism. In such networks, congestion control of the shared channel is fundamental in order to support regular and timely delivery of time-critical data. The obvious approach towards avoiding congestion is to reduce the sending rate of update messages. However, this could lead to outdated information, impairing the proper functioning of applications depending on it.

The trade-off between congestion of the communication channel and message update frequency is captured by a specific metric, the Age of Information (AoI) [1]. Let $u_{i j}(t)$ be the time when the last update message coming from node $i$ is received by node $j$ before time $t$. The AoI of node $i$ 's data stored at node $j$ at time $t$ is defined as $H_{i j}(t)=t-u_{i j}(t)$. This definition assumes the data has age 0 when it arrives at node $i$.

The AoI concept is reviewed extensively in [1]. Liu and Bennis [2] address a centralized industrial sensor network and optimize scheduling of data collection to set a bound on the maximal AoI. They estimate the tail of the maximal AoI distribution via Generalized Extreme Value theory. A detailed analysis of AoI in queuing systems with First Come First Served (FCFS) and Last Come First Served (LCFS) disciplines

A. Baiocchi is with the Department of Information Engineering, Electronics and Telecommunications (DIET), University of Rome Sapienza, Italy, e-mail: andrea.baiocchi@uniroma1.it.

Ion Turcanu is with the Interdisciplinary Centre for Security, Reliability and Trust (SnT), University of Luxembourg, e-mail: ion.turcanu@uni.lu. is presented in [3]. The authors give a general formula for the probability distribution of AoI for a $G / G / 1$ queue and then specialize the result for various instances of queues. Optimal queuing policies to minimize AoI in a single-hop queue are also investigated by Bedewy et al. [4]. In another study, Bedewy et al. [5] consider the problem of minimizing the AoI while reducing the energy consumption in a network of low-power nodes via a sleep-wake scheduling mechanism. Kadota et al. [6], [7] provide models to assess optimal scheduling and define heuristics to minimize AoI in a centralized scenario where a base station collects data from a population of terminals. Sun et al. [8] study optimal scheduling to minimize the AoI of updates sent from a source node to a destination via a channel. Yates and Kaul [9] derive the AoI metric for a slotted-ALOHA network in which wireless nodes send periodic updates to a sink node. All these works have a common feature: they focus on a specific class of networks in which nodes send periodic updates to a central repository/sink. We consider instead a vehicular network, in which every node is both a transmitter and receiver of update messages to/from their one-hop neighbors.

Several works have studied the AoI in CSMA-based networks. Kaul et al. [10] design an adaptive beaconing strategy for vehicular networks that minimizes the AoI by balancing the load on the wireless access network and the frequency of updates. Gopal and Kaul [11] propose a model that characterizes the AoI of a DSRC node and study the coexistence of DSRC and Wi-Fi nodes in the same frequency band. In a recent work, Maatouk et al. [12] study the problem of finding the optimal back-off time for links in a CSMA network in order to minimize the average AoI. These works make an important assumption: all nodes are able to hear each other, i.e., the hidden node problem does not exist.

The core contribution of this work is a model for the calculation of the AoI in a distributed update message exchange system, based on a CSMA network with partial sensing (i.e., we consider the hidden node problem in our model). In our previous work [13] we provided a first attempt towards modeling such a network. The model we propose in this work improves over the one elaborated in [13] in two ways: it is simpler and more accurate. The main ideas that allowed for this simplification stem from (i) realizing that in vehicular networks the message generation period is typically much larger than the mean access time, and (ii) introducing a simple queueing model, the $M / D / \infty$ queue, to account for the effect of partial sensing and the ensuing duration of the busy air time interval beyond the strict frame transmission time. In the following, we develop the analytical model (Section II), validate it against simulations and evaluate the system performance (Section III). We present the concluding remarks in Section IV. 


\section{AnAlytical MODEL FOR AoI EVAluation}

\section{A. Access protocol}

We consider a set of $n$ nodes exchanging one-hop messages. As soon as a new message is generated, the node starts the channel access procedure, eventually transmitting the message as a broadcast frame. Channel access is operated according to IEEE 802.11p MAC protocol. A backlogged node waits for the channel to stay idle for a DIFS time, then it selects a random back-off counter uniformly distributed between 1 and $W_{0}$, the contention window size. If the node senses an idle channel within a back-off slot time, it decrements its counter, otherwise it freezes the counter, waiting for the channel to become idle again for a DIFS time. When the counter hits 0 , the node transmits. No ACK or re-transmission is provided for, since only broadcast frames are used.

\section{B. Assumptions}

Node interaction is modeled by means of a contact graph. Let $\mathbf{A}$ denote the adjacency matrix and $a_{i j}$ an element of this matrix, where $a_{i j}=1$ if node $j$ can decode messages from node $i$, and 0 otherwise. We assume that the reception of a frame at node $i$ is disrupted if and only if any neighbor of $i$ overlaps its own transmission with the ongoing frame reception at $i$. In other words, the contact graph also describes the potentially disruptive interference.

We also assume that messages have a constant length and all nodes use the same air bit rate. Let $T$ denote the constant frame transmission time, including DIFS and any overhead. New messages are generated periodically with period $D_{i}$ at node $i$. While engaged in the access procedure, a node only stores up to one single further message (the most recent), if any arrives. The following assumptions are key to simplifying the model derivation: (i) the states of nodes are independent of one another; (ii) the duration of a busy air time interval sensed by a node (i.e., the channel busy time elapsing between two consecutive back-off counter decrements) is modeled as the busy period of an $M / G / \infty$ queue; (iii) the generation period $D_{i} \gg \mathrm{E}\left[C_{i}\right]$, where $C_{i}$ denotes the time required to complete channel access ${ }^{1}$.

\section{Model analysis}

Let us consider a tagged node $i$ sending a message at time $t_{k}^{(i)}, k \in \mathbb{Z}$. We let $Y_{i}(k)=t_{k}^{(i)}-t_{k-1}^{(i)}$ be the $k$-th interdeparture time of the message queued at node $i$. Since a station engaged in the access procedure of its current frame only stores the most recent new arrival, if any, we have $Y_{i}(k)=$ $\max \left\{0, D_{i}-C_{i}(k-1)\right\}+C_{i}(k)$, where $C_{i}(k)$ is the time required to complete the access operation for frame $k$. The first term accounts for the time elapsing since the departure of frame $k-1$ on the PHY channel and the arrival of frame $k$ from the application. Figure 1 illustrates the derivation of $Y_{i}(k)$. In particular, when $D_{i}>C_{i}(k-1)$ (Figure 1a), we have

\footnotetext{
${ }^{1}$ This last assumption is consistent with the message generation periods in vehicular networks - which range between $0.1-1 \mathrm{~s}-$ as stated in ETSI EN 302 637-2.
}

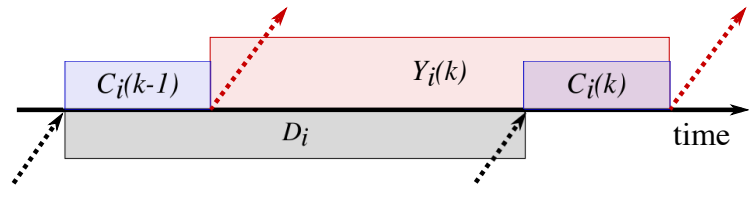

(a) $D_{i}>\mathrm{E}\left[C_{i}\right]$

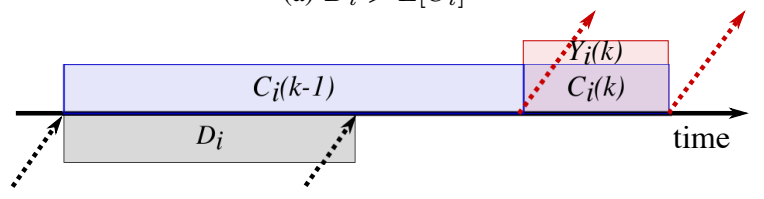

(b) $D_{i}<\mathrm{E}\left[C_{i}\right]$

Figure 1. Message inter-departure time $Y(k)$. Black dotted arrows indicate arrival of messages from the application. Red dotted arrows show sending times of messages on the wireless channel.

$Y_{i}(k)=D_{i}-C_{i}(k-1)+C_{i}(k)$, while for $D_{i} \leq C_{i}(k-1)$ (Figure 1b) we have $Y_{i}(k)=C_{i}(k)$.

The time $Z_{i j}$ to deliver a new message from $i$ to $j$ is

$$
Z_{i j}=\sum_{k=1}^{N_{i j}} Y_{i}(k)
$$

where $Y_{i}(k) \sim Y_{i}$ and $N_{i j}$ is the number of attempts required to make a successful message delivery from $i$ to $j$. Assuming that successive message transmission attempts are independent of one another, $N_{i j}$ has a geometric probability distribution:

$$
\mathcal{P}\left(N_{i j}=h\right)=P_{s, i j}\left[1-P_{s, i j}\right]^{h-1}, \quad h \geq 1 .
$$

where $P_{s, i j}$ is the probability of a successful message delivery from node $i$ to node $j$, defined in Equation (19).

The mean value of the AoI from $i$ to $j, H_{i j}$, is akin to the mean remaining service time in a queue, i.e.

$$
\mathrm{E}\left[H_{i j}\right]=\frac{\mathrm{E}\left[Z_{i j}^{2}\right]}{2 \mathrm{E}\left[Z_{i j}\right]}=\frac{\mathrm{E}\left[Y_{i}^{2}\right]}{2 \mathrm{E}\left[Y_{i}\right]}+\mathrm{E}\left[Y_{i}\right]\left(\frac{1}{P_{s, i j}}-1\right)
$$

The expression above allows computation of the mean AoI of messages flowing from $i$ to $j$. The AoI at $j$ can be obtained by averaging over all neighbor nodes of $j$. If $j$ is isolated, it receives no message, so AoI is meaningless. Apart from this marginal case, we define

$$
\mathrm{E}\left[H_{j}\right]=\frac{\sum_{i=1}^{n} a_{i j} \mathrm{E}\left[H_{i j}\right]}{\sum_{i=1}^{n} a_{i j}}
$$

provided that the number of node $j$ 's neighbors $n_{j} \equiv$ $\sum_{i=1}^{n} a_{i j}>0$. The overall average AoI of the entire network can be summarized by the following definition:

$$
\mathrm{E}[H]=\sum_{j=1}^{n} \frac{n_{j}}{n} \mathrm{E}\left[H_{j}\right]=\frac{1}{n} \sum_{j=1}^{n} \sum_{i=1}^{n} a_{i j} \mathrm{E}\left[H_{i j}\right]
$$

where $n=n_{1}+\cdots+n_{n}=\sum_{j=1}^{n} \sum_{i=1}^{n} a_{i j}$.

To evaluate the mean AoI, it remains to find expressions for $P_{s, i j}$ and for the first two moments of $Y_{i}$. Let us start with the moments of $Y_{i}$.

Since we assume $D_{i} \gg \mathrm{E}\left[C_{i}\right]$, we have $Y_{i}(k) \approx D_{i}-$ $C_{i}(k-1)+C_{i}(k)$ with high probability. At equilibrium, we have $Y_{i}(k) \sim Y_{i}$ and $C_{i}(k) \sim C_{i}$, hence

$$
\mathrm{E}\left[Y_{i}\right] \approx D_{i} \quad \sigma_{Y_{i}}^{2} \approx 2 \sigma_{C_{i}}^{2}
$$


This is a consequence of $D_{i}$ being a constant and of the independence approximation, whereby $C_{i}(k)$ and $C_{i}(k-1)$ are i.i.d. random variables. As for $C_{i}$, this is the sum of the back-off time $B_{i}$ plus the transmission time $T$ :

$$
C_{i}=T+B_{i}=T+\sum_{h=1}^{M} X_{i}(h)
$$

where $M$ is a uniform integer random variable over the range $\left\{1, \ldots, W_{0}\right\}$, and $X_{i}(h) \sim X_{i}$ are i.i.d. random variables. Here, $X_{i}$ denotes the steady state random variable defined as the time interval between two consecutive back-off counter decrements at node $i$. It is easy to find from Equation (7) that:

$$
\sigma_{C_{i}}^{2}=\frac{W_{0}^{2}-1}{12}\left(\mathrm{E}\left[X_{i}\right]\right)^{2}+\frac{W_{0}-1}{2} \sigma_{X_{i}}^{2}
$$

The calculation of the second moment of $C_{i}$, and hence of $Y_{i}$, reduces to finding expressions for the first two moments of the random variable $X_{i}$. We have

$$
X_{i}= \begin{cases}\delta & \text { w.p. } q_{i} \\ V_{i}+\delta & \text { w.p. } 1-q_{i}\end{cases}
$$

where $\delta$ is the back-off slot time, $q_{i}$ is the probability that no neighbor of node $i$ starts transmitting, and $V_{i}$ is the duration of the busy air time interval as seen by node $i . V_{i}$ does not reduce to the transmission time $T$, since nodes are not synchronized by channel sensing, given that each node senses only a small fraction of all $n$ nodes. Therefore, if a neighbor of $i$, say $j$, starts transmitting, it can happen that another neighbor of $i$, say $k$, which does not hear the on-going transmission of $j$, can initiate a new transmission during $j$ 's transmission.

With standard probabilistic arguments it can be found from Equation (9) that:

$$
\begin{aligned}
& \mathrm{E}\left[X_{i}\right]=\delta+\left(1-q_{i}\right) \mathrm{E}\left[V_{i}\right] \\
& \sigma_{X_{i}}^{2}=\delta^{2}+2 \delta\left(1-q_{i}\right) \mathrm{E}\left[V_{i}\right]+\left(1-q_{i}\right) \mathrm{E}\left[V_{i}^{2}\right]
\end{aligned}
$$

All we need to compute these expressions are the probabilities $q_{i}$ and the first two moments of the random variables $V_{i}$.

Thanks to the independence assumption, the probability $q_{i}$ for a tagged node $i$ is given by

$$
q_{i}=\prod_{j}\left(1-a_{i j} \tau_{j}\right)
$$

where $\tau_{j}$ is the probability that node $j$ starts transmitting, after having sensed an idle back-off time slot.

At steady-state, node $i$ is transmitting with probability $p_{\mathrm{tx}, i}=$ $T / \mathrm{E}\left[Y_{i}\right]$, is backlogged and counting down with probability $p_{\mathrm{bo}, i}=\mathrm{E}\left[B_{i}\right] / \mathrm{E}\left[Y_{i}\right]$, and is idle with probability $p_{\text {idle }, i}=$ $1-p_{\mathrm{tx}, i}-p_{\mathrm{bo}, i}$. Given that node $i$ is backlogged, the probability of transmission is $\mathrm{E}\left[X_{i}\right] / \mathrm{E}\left[B_{i}\right]$, which is found by applying the renewal reward theorem. Taking into account that node $i$ can start a new transmission only when it is backlogged, the probability that it starts a new transmission, given that it is not already transmitting, is

$$
\tau_{i}=\frac{\left(1-p_{\mathrm{bo}, i}\right) \cdot 0+p_{\mathrm{bo}, i} \cdot \frac{\mathrm{E}\left[X_{i}\right]}{\mathrm{E}\left[B_{i}\right]}}{1-p_{\mathrm{tx}, i}}=\frac{\mathrm{E}\left[X_{i}\right]}{\mathrm{E}\left[Y_{i}\right]-T}
$$

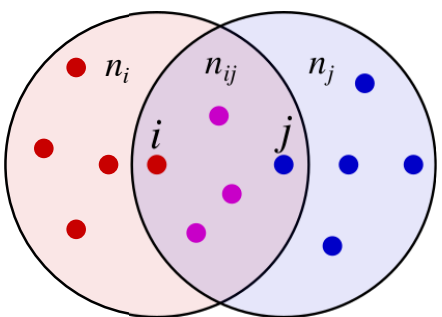

Figure 2. An illustration of two nodes with partially overlapping neighbors.

As for the busy air time $V_{i}$ sensed by node $i$, in the following we identify $V_{i}$ as the busy period of an $M / D / \infty$ queue, with service time equal to $T$, the frame transmission time. This is a simple way to capture the fact that neighbors of node $i$ can start new transmissions even if a neighbor of $i$ is already transmitting, due to the partial sensing among nodes of the considered CSMA network. In general, it is $V \geq T$. We will define the model of $V$ so that $V=T$ with probability 1 in case all nodes can sense each other.

The Laplace transform of the Probability Density Function (PDF) of $V_{i}$ is [14]

$$
\varphi_{V_{i}}(s)=\frac{s+\nu_{i}}{s e^{s T+\nu_{i} T}+\nu_{i}}
$$

where $\nu_{i}$ denotes the mean arrival rate of the $M / D / \infty$ queue. The first two moments of $V_{i}$ are given by

$$
\mathrm{E}\left[V_{i}\right]=T \frac{e^{b_{i}}-1}{b_{i}} \quad \mathrm{E}\left[V_{i}^{2}\right]=T^{2} \frac{2 e^{b_{i}}}{b_{i}}\left(\frac{e^{b_{i}}-1}{b_{i}}-1\right)
$$

where $b_{i}=\nu_{i} T$.

The mean arrival rate $\nu_{i}$ associated with node $i$ is identified as follows. The mean number of customers served in a busy period of the $M / D / \infty$ queue is $e^{\nu_{i} T}$. The mean number of transmissions of the neighbors of $i$, during a single busy air time interval, is

$$
1+\psi_{i} \mathrm{E}\left[V_{i}\right] \sum_{j} a_{i j} \frac{1}{\mathrm{E}\left[Y_{j}\right]}=e^{\nu_{i} T}
$$

where the factor $\psi_{i}$ accounts for the fact that neighbors of $i$ interact among themselves via carrier sensing, and hence some of them are blocked by other neighbors' transmissions due to carrier sensing. Using the first moment of $V_{i}$, defined in Equation (15), we find:

$$
\nu_{i}=\psi_{i} \sum_{j} a_{i j} \frac{1}{\mathrm{E}\left[Y_{j}\right]}
$$

The busy air time is started by one or possibly more nodes. Neighbors of $i$ that are also neighbors of the nodes starting the busy air time cannot transmit, due to the rule "listen-beforetalk". However, nodes that are not neighbors of the nodes that started the busy air time could possibly intervene during the busy air time. Let $n_{i j}$ denote the number of nodes that are common neighbors of $i$ and $j$, as shown in Figure 2. If $j$ starts transmitting, it holds a fraction $n_{i j} / n_{i}$ of the $n_{i}$ neighbors of $i$. The average fraction of nodes that are not affected by $j$ is $1-\tau_{j} n_{i j} / n_{i}$. Given that at least one node transmits, the 
average fraction of nodes that are not affected by those that start the busy air time is

$$
\psi_{i}=\frac{\prod_{j}\left(1-a_{i j} \tau_{j} n_{i j} / n_{i}\right)-\prod_{j}\left(1-a_{i j} \tau_{j}\right)}{1-\prod_{j}\left(1-a_{i j} \tau_{j}\right)}
$$

Note that, in the case that all nodes can hear (the graph is a full mesh), it turns out that $\psi_{i}=0$, hence $\nu_{i}=0$ and $V_{i}=T$.

According to Equations (6), (10) and (13), we have $\tau_{i} \approx\left(\delta+\left(1-q_{i}\right) \mathrm{E}\left[V_{i}\right]\right) /\left(D_{i}-T\right)$. Both $q_{i}$ and $\mathrm{E}\left[V_{i}\right]$ are determined once the values of the $\tau_{i}$ 's are given. We see therefore that the numerical evaluation of the model requires the solution of a fixed point equation system of the kind $\tau_{i}=F_{i}\left(\tau_{1}, \ldots, \tau_{n}\right), i=1, \ldots, n$. Since the $F_{i}$ 's are continuous functions, Brouwer's theorem guarantees that there exists a fixed point in the hypercube $\left[\tau_{1}, \ldots, \tau_{n}\right] \in[0,1]^{n}$.

Now we turn to the derivation of an expression for $P_{s, i j}$, the probability of successful message delivery from $i$ to $j$. Let us focus on a transmitting node $i$ and one of its neighbors, say node $j$. The frame from $i$ is received successfully by $j$ if no other neighbor of $j$ transmits an overlapping frame at the same time as $i$. We distinguish nodes that are neighbors of both $i$ and $j$, which are conditioned by node $i$ 's transmission, and nodes that are neighbors of $j$, but not of $i$. The probability of a successful message delivery from $i$ to $j$ is:

$$
P_{s, i j}=\left(1-\tau_{j}\right) \prod_{k \neq i, j}\left(1-a_{k j} a_{k i} \tau_{k}\right)\left(1-a_{k j}\left(1-a_{k i}\right) \theta_{k}\right)
$$

where $\theta_{k} \approx 2 T / D_{k}$. The approximation follows from the fact that a node $k$ (neighbor of $j$, but not of $i$ ), whose time evolution is not conditioned by node $i$, can collide with a transmission of $i$ starting at time $t_{i}$ only if its own transmission starts at $t_{k} \in\left[t_{i}-T, t_{i}+T\right)$. Since $k$ sends out one message every $D_{k}$ on average, the probability that $k$ hits $i$ 's transmission can be expressed as $2 T / D_{k}$.

\section{MOdEL VALIDATION AND APPLICATION}

We apply the model to an IEEE 802.11p VANET. The graph is obtained by running realistic simulations in urban environments using Veins framework [15]. For the communication channel simulation, we use the Two-Ray Interference and Simple Obstacle Shadowing models provided by Veins. Every vehicle records the received neighbor information in its local neighbor table. Neighbors of a node correspond to those listed in the neighbor table. The adjacency matrix $\mathbf{A}$ is created by taking a snapshot of the local neighbor tables for all the vehicles in the simulation. We set $D_{i}=D_{0}$ for every node $i$. The message sending period $D_{0}$ is used as a variable, ranging from $50 \mathrm{~ms}$ up to $1000 \mathrm{~ms}$, and the average AoI is evaluated for a given message size $L$. We set $L=1000$ Byte. By assuming an air bit rate of $6 \mathrm{Mbit} / \mathrm{s}$, we have $T=2.85 \mathrm{~ms}$ for $L=1000$ Byte. Other MAC parameters are $\delta=0.013 \mathrm{~ms}$ and $W_{0}=16$.

Figure 3 compares the average AoI values as a function of the sending period $D_{0}$ for three scenarios: Cologne [16], Luxembourg [17] (real maps) and Manhattan Grid road network (artificial). The last one is considered for two different message sizes, 1000 Byte and 500 Byte. We compare the performance

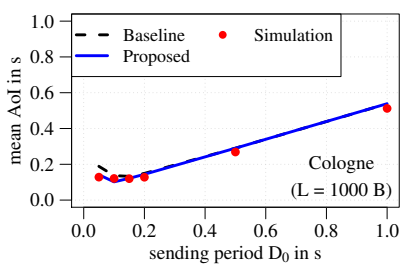

(a)

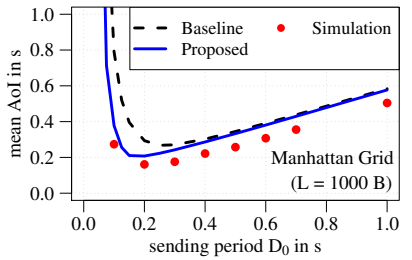

(c)

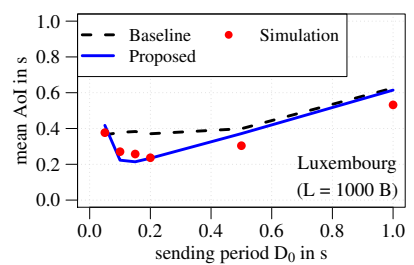

(b)

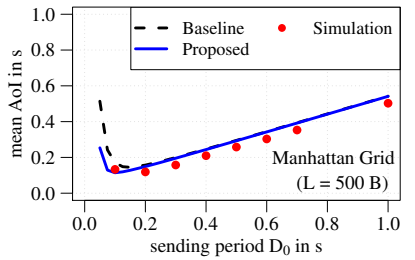

(d)
Figure 3. Mean AoI, analytical model vs simulations: (a) Cologne, (b) Luxembourg, (c) Manhattan Grid [ $L=1000$ Byte], and (d) Manhattan Grid $[L=500$ Byte $]$

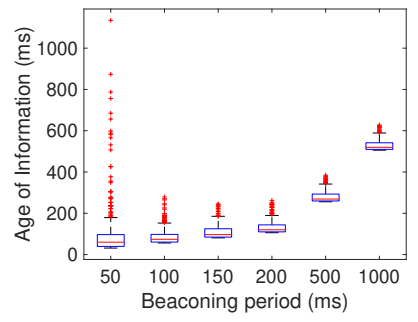

(a)

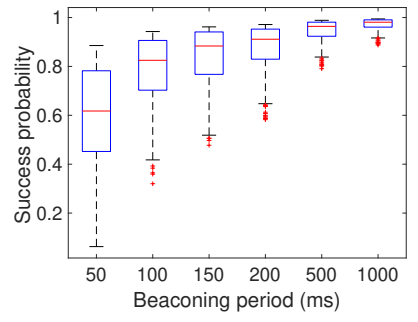

(c)

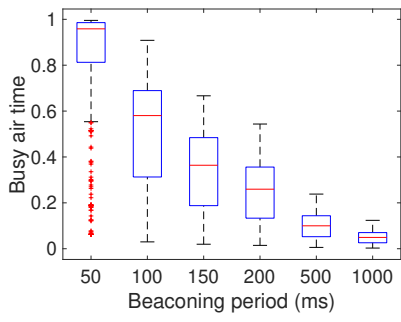

(b)

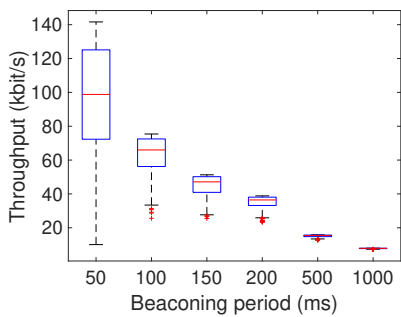

(d)
Figure 4. Performance spread as a function of the message sending period for the Cologne dataset: (a) average AoI; (b) average busy air time; (c) probability of successful reception; (d) effective throughput.

of the proposed analytical model with the one described in [13], denoted as "Baseline" in the figure, as well as with the results obtained from the realistic simulations. It is apparent that the model yields a good approximation, somewhat overestimating the actual AoI in case of Manhattan grid (Figure 3c and Figure 3d), especially for large message sizes. Also, the proposed model is more accurate when compared to the Baseline solution, particularly for low values of $D_{0}$, where the channel is more congested.

The overall average performance does not reveal the spread of performance achieved by different nodes. To highlight this aspect, we consider the boxplot of four metrics, for the Cologne dataset, illustrated in Figure 4. The four considered metrics are: (i) the average AoI of the data stored by each node (Figure 4a); (ii) the average coefficient of utilization of the radio channel 


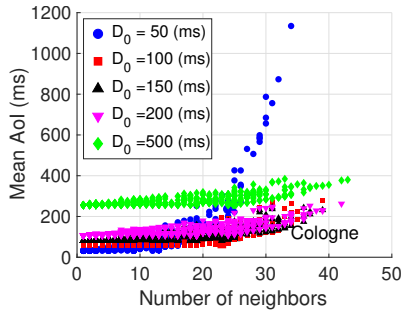

(a)

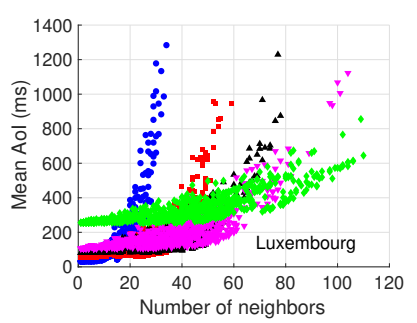

(c)

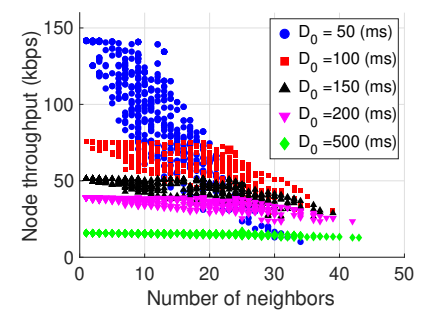

(b)

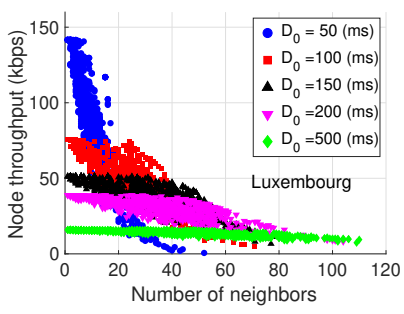

(d)
Figure 5. Performance spread as a function of the number of neighbors: average AoI (left) and effective throughput (right); Cologne (top) and Luxembourg (bottom).

sensed by each node (Figure 4b): for node $i$ it is $\rho_{i}=(1-$ $\left.q_{i}\right) \mathrm{E}\left[V_{i}\right] / \mathrm{E}\left[X_{i}\right]$; (iii) the probability of realizing a successful message reception (Figure 4c); (iv) the effective throughput, i.e., the delivered data rate per node (Figure 4d): this is obtained for node $i$ as $\Lambda_{i}=L \sum_{j} a_{i j} P_{s, i j} / D_{i}$.

The AoI per node exhibits a decreasing spread for increasing values of $D_{0}$. What is more, it has a large number of outliers for short sending periods. This means that a significant fraction of nodes has a quite large value of AoI, departing strongly from the system average. The same phenomenon is also highlighted by the other metrics. Each of these has a large spread and possibly a large number of outliers for small values of $D_{0}$, while performance become more regular as $D_{0}$ grows.

Figure 5 shows scatter plots that highlight the correlation of performance spread with the number of neighbors of each node for two key metrics: the average AoI per node and the effective throughput per node.

Both AoI and throughput exhibit a clear correlation with the number of neighbors. Nodes operating in crowded areas can depart strongly from average system performance, experiencing a significant worsening of their performance metrics. The spread of performance values narrows as the sending interval $D_{0}$ increases. Operating around $D_{0} \approx 200 \mathrm{~ms}$ not only leads to system AoI minimization, but results in a greater fairness among nodes.

\section{CONCLUSION}

We have addressed a network of agents sending periodic update messages to their neighbors on a regular basis and developed a model to predict the AoI metric of the agent network. The model lends itself to describing one-hop broadcast message traffic in CSMA networks with generally partial sensing. As for the AoI, it appears to be reasonably accurate, at least as long as we consider the contact-graph model as representative of neighborhood relationships among nodes (reachability and interference). The outcome of our analysis, supported by contact graphs based on realistic vehicular simulations, is that our model is able to capture the general trade-off between AoI, channel load and fairness among nodes. In addition, the model allows investigation of the AoI performance as seen by each node, not just as a system average, providing a more granular view of the system performance.

A future improvement of the model could be to relax the assumption $D_{i} \gg \mathrm{E}\left[C_{i}\right]$. This can be done starting from the analysis presented in this paper and going through the numerical inversion of the Laplace transforms of PDFs to calculate the moments of the non-linearity of $\max \left\{0, D_{i}-C_{i}(k-1)\right\}$. In addition, the model could be developed into a queueing network over a CSMA network to offer a powerful analysis tool for multi-hop dissemination protocols, i.e., message spreading not limited to a single hop.

\section{REFERENCES}

[1] A. Kosta, N. Pappas, V. Angelakis, et al., "Age of information: A new concept, metric, and tool," Foundations and Trends® in Networking, vol. 12, no. 3, pp. 162-259, 2017.

[2] C.-F. Liu and M. Bennis, "Taming the Tail of Maximal Information Age in Wireless Industrial Networks," IEEE Communications Letters, vol. 23, no. 12 , pp. 2442-2446, Dec. 2019.

[3] Y. Inoue, H. Masuyama, T. Takine, and T. Tanaka, "A General Formula for the Stationary Distribution of the Age of Information and Its Application to Single-Server Queues," IEEE Transactions on Information Theory, vol. 65, no. 12, pp. 8305-8324, Dec. 2019.

[4] A. M. Bedewy, Y. Sun, and N. B. Shroff, "Minimizing the Age of Information Through Queues," IEEE Transactions on Information Theory, vol. 65, no. 8, pp. 5215-5232, Aug. 2019.

[5] A. M. Bedewy, Y. Sun, R. Singh, and N. B. Shroff, "Optimizing information freshness using low-power status updates via sleep-wake scheduling," arXiv preprint arXiv:1910.00205, 2019.

[6] I. Kadota and E. Modiano, "Minimizing the age of information in wireless networks with stochastic arrivals," IEEE Transactions on Mobile Computing, Dec. 2019

[7] I. Kadota, A. Sinha, and E. Modiano, "Scheduling Algorithms for Optimizing Age of Information in Wireless Networks With Throughput Constraints," IEEE/ACM Transactions on Networking, vol. 27, no. 4, pp. 1359-1372, Aug. 2019.

[8] Y. Sun, E. Uysal-Biyikoglu, R. D. Yates, C. E. Koksal, and N. B. Shroff, "Update or Wait: How to Keep Your Data Fresh," IEEE Transactions on Information Theory, vol. 63, no. 11, pp. 7492-7508, Nov. 2017.

[9] R. D. Yates and S. K. Kaul, "Status updates over unreliable multiaccess channels," in IEEE International Symposium on Information Theory (ISIT), IEEE, Jun. 2017, pp. 331-335.

[10] S. Kaul, M. Gruteser, V. Rai, and J. Kenney, "Minimizing age of information in vehicular networks," in 8th Annual IEEE Communications Society Conference on Sensor, Mesh and Ad Hoc Communications and Networks, Jun. 2011, pp. 350-358.

[11] S. Gopal and S. K. Kaul, "A game theoretic approach to DSRC and WiFi coexistence," in IEEE Conference on Computer Communications Workshops (INFOCOM WKSHPS), IEEE, Apr. 2018, pp. 565-570.

[12] A. Maatouk, M. Assaad, and A. Ephremides, "On the Age of Information in a CSMA Environment," IEEE/ACM Transactions on Networking, vol. 28 , no. 2, pp. 818-831, 2020.

[13] A. Baiocchi and I. Turcanu, "A Model for the Optimization of Beacon Message Age-of-Information in a VANET," in 29th International Teletraffic Congress (ITC 29), vol. 1, Genoa, Italy: IEEE, Sep. 2017, pp. $108-116$.

[14] L. Liu and D.-H. Shi, "Busy period in $G I^{X} / G / \infty$," Journal of Applied Probability, pp. 815-829, 1996.

[15] C. Sommer, R. German, and F. Dressler, "Bidirectionally Coupled Network and Road Traffic Simulation for Improved IVC Analysis," IEEE Trans. on Mobile Computing, vol. 10, no. 1, pp. 3-15, Jan. 2011

[16] S. Uppoor, O. Trullols-Cruces, M. Fiore, and J. M. Barcelo-Ordinas, "Generation and analysis of a large-scale urban vehicular mobility dataset," IEEE Transactions on Mobile Computing, vol. 13, no. 5, pp. 1061-1075, 2013

[17] L. Codeca, R. Frank, S. Faye, and T. Engel, "Luxembourg SUMO traffic (LuST) scenario: Traffic demand evaluation," IEEE Intelligent Transportation Systems Magazine, vol. 9, no. 2, pp. 52-63, 2017. 